\title{
The $n$-card problem, stochastic matrices, and the Extreme Principle
}

\author{
Justin H.C. Chan Jonathan Jedwab* \\ Department of Mathematics \\ Simon Fraser University \\ Burnaby, BC, Canada \\ $\{$ jhc34,jed\}@sfu.ca
}

Submitted: Jan 16, 2012; Accepted: Jun 17, 2012; Published: Jun 28, 2012

Mathematics Subject Classifications: 05A05

\begin{abstract}
The $n$-card problem is to determine the minimal intervals $[u, v]$ such that for every $n \times n$ stochastic matrix $A$ there is an $n \times n$ permutation matrix $P$ (depending on $A)$ such that $\operatorname{tr}(P A) \in[u, v]$. This problem is closely related to classical mathematical problems from industry and management, including the linear assignment problem and the travelling salesman problem. The minimal intervals for the $n$-card problem are known only for $n \leqslant 4$.

We introduce a new method of analysis for the $n$-card problem that makes repeated use of the Extreme Principle. We use this method to answer a question posed by Sands (2011), by showing that $[1,2]$ is a solution to the $n$-card problem for all $n \geqslant 2$. We also show that each closed interval of length $\frac{n}{n-1}$ contained in $[0,2)$ is a solution to the $n$-card problem for all $n \geqslant 2$.
\end{abstract}

Keywords: stochastic matrix; permutation matrix; transversal sum; trace; Extreme Principle; $n$-card problem

\section{Introduction}

Let $n \geqslant 2$ be an integer. An $n \times n$ stochastic matrix is an $n \times n$ matrix $\left(a_{i j}\right)$ of non-negative real numbers, each of whose row sums is 1 . A transversal sum of $\left(a_{i j}\right)$ is a sum of the form $\sum_{i=1}^{n} a_{\sigma(i), i}$, for some permutation $\sigma$ of $\{1,2, \ldots, n\}$. A solution to the $n$-card problem is an interval $[u, v]$ such that every $n \times n$ stochastic matrix contains at least one transversal sum in $[u, v]$. Equivalently, a solution to the $n$-card problem is an interval $[u, v]$ such that

*Supported by NSERC. 
for every $n \times n$ stochastic matrix $A$ there is an $n \times n$ permutation matrix $P$ such that $\operatorname{tr}(P A) \in[u, v]$. We wish to determine the minimal solutions to the $n$-card problem for each $n$, namely those solutions $[u, v]$ for which no proper subinterval of $[u, v]$ is a solution.

The $n$-card problem is closely related to well-known mathematical problems with industrial and management applications, involving the possible values of $\operatorname{tr}(P A)$ for a permutation matrix $P$ and a fixed square matrix $A$. In particular, the linear assignment problem, "one of the most famous problems in linear programming and in combinatorial optimization [BDM09]," is to minimize $\operatorname{tr}(P A)$ over all permutation matrices $P$ (see [BDM09, Chapter 4] for a detailed historical account of the development of algorithms for its solution, and an equivalent formulation in terms of weighted bipartite matchings); the travelling salesman problem is the special case in which the permutation corresponding to $P$ is cyclic [Flo56].

The terminology of the $n$-card problem reflects its original formulation [San01], [LS05] involving a set of $n$ cards, each containing $n$ non-negative real numbers written in a row and summing to 1, with the transversal sum representing the diagonal sum formed when the cards are placed one below the other according to some permutation. In 2001, Sands [San01] asked for a proof that $\left[\frac{1}{2}, \frac{3}{2}\right]$ is a solution to the 3 -card problem. Lenza and Sands [LS05] introduced the generalization to the $n$-card problem in 2005.

The interval $[0,1]$ is a minimal solution to the $n$-card problem for all $n \geqslant 2$ [San11, Lemma 3], but it is not the only minimal solution. Indeed, it is easily checked by hand that the minimal solutions to the 2-card problem are

$$
[0,1], \quad[1,2] .
$$

The minimal solutions to the 3-card problem are [LS05]

$$
[0,1], \quad\left[\frac{1}{2}, \frac{3}{2}\right], \quad[1,2],
$$

and the minimal solutions to the 4-card problem are [LS05], [San11]

$$
[0,1], \quad\left[\frac{1}{3}, \frac{4}{3}\right], \quad\left[\frac{2}{3}, \frac{5}{3}\right], \quad[1,2] .
$$

The method of [LS05] and [San11] is to establish a particular interval $[u, v]$ as a solution to the $n$-card problem (for $n=3$ or 4 ), by using intersecting permutations to show that the number of transversal sums greater than $v$, plus the number of transversal sums less than $u$, is always less than $n$ !. The method has two drawbacks: it relies on a laborious case analysis for $n=4$, and does not extend to $n \geqslant 5$ [LS05, p.6].

In this paper we introduce a new method for analysing the $n$-card problem that makes repeated use of the Extreme Principle [Zei07]. We believe that this method could shed light on other problems involving $\operatorname{tr}(P A)$, where $P$ is a permutation matrix and $A$ is a fixed square matrix. The Extreme Principle directs attention to the "largest" and "smallest" elements of a problem. In the present context, we assume for a contradiction that no transversal sum of an $n \times n$ stochastic matrix lies in some interval $[u, v]$, and then consider the smallest transversal sum $d$ greater than $v$. Then, if a transversal sum is less than $d$, it must be less than $u$. We seek such transversal sums, involving exactly $n-2$ of 
the original summands of $d$, in order to reach a contradiction. We thereby obtain strong new restrictions for all $n \geqslant 5$. In particular, we solve Problem 5 of [San11] as follows.

Theorem 1. For all $n \geqslant 2$, the interval $[1,2]$ is a solution to the $n$-card problem.

We also prove the following result.

Theorem 2. For all $n \geqslant 2$, each closed interval of length $\frac{n}{n-1}$ contained in $[0,2)$ is a solution to the $n$-card problem.

The length $\frac{n}{n-1}$ in Theorem 2 is the smallest possible for a general interval, because the $n \times n$ stochastic matrix having one row $\left(0, \frac{1}{n-1}, \frac{1}{n-1}, \ldots, \frac{1}{n-1}\right)$ and $n-1$ rows $(1,0,0, \ldots, 0)$ has transversal sums of 0 and $\frac{n}{n-1}$ only.

On the other hand, the known complete set of minimal solutions (2) for $n=3$ and (3) for $n=4$ shows that the interval length in Theorem 2 can be reduced to 1 for specific intervals. By reference to particular $n \times n$ stochastic matrices, Sands [San11] showed that every solution to the $n$-card problem for $n \geqslant 2$ must contain a length 1 interval $\left[\frac{k}{n-1}, 1+\frac{k}{n-1}\right]$ for some $k \in\{0,1, \ldots, n-1\}$. Problem 3 of [San11] asks whether each such length 1 interval is itself a solution to the $n$-card problem, which would imply that the complete set of minimal solutions to the $n$-card problem comprises these $n$ intervals. This question remains open for $n>4$.

\section{The interval $[1,2]$}

In this section we prove Theorem 1 . We firstly establish some preliminary lemmas.

Lemma 3. Let $\left(a_{i j}\right)$ be an $n \times n$ stochastic matrix, all of whose transversal sums lie outside an interval $[u, v]$ containing 1 . Then $\left(a_{i j}\right)$ has at least one transversal sum less than $u$, and at least one transversal sum greater than $v$.

Proof. Each entry of $\left(a_{i j}\right)$ is contained in exactly $(n-1)$ ! transversal sums, so the mean of all transversal sums is $(n-1) !\left(\sum_{i, j} a_{i j}\right) / n !=1$. Therefore at least one transversal sum is at most 1 , and so by assumption less than $u$. Similarly, at least one transversal sum is greater than $v$.

An immediate consequence of Lemma 3 is that, as noted earlier, $[0,1]$ is a solution to the $n$-card problem for all $n \geqslant 2$.

The rows and columns of an $n \times n$ stochastic matrix can be permuted without changing the set of its $n$ ! transversal sums. Our method relies on examining the effect of transposing two rows of an $n \times n$ stochastic matrix, and thereby bounding the matrix entries. We now show that if an $n \times n$ stochastic matrix has a sufficiently large diagonal sum then there must be a transposition of two rows that decreases this diagonal sum. We prove this result for the following slightly more general case of an $n \times n$ substochastic matrix (each of whose row sums is at most 1). 
Lemma 4. Let $\left(a_{i j}\right)$ be an $n \times n$ substochastic matrix. Suppose $\left(a_{i j}\right)$ has diagonal sum greater than 1 . Then

$$
a_{k k}+a_{\ell \ell}>a_{k \ell}+a_{\ell k} \text { for some } k, \ell \text {. }
$$

Proof. Suppose, for a contradiction, that $a_{i i}+a_{j j} \leqslant a_{i j}+a_{j i}$ for all $i, j$. Sum this inequality over all $i, j$ to obtain $2 n \sum_{i} a_{i i} \leqslant 2 \sum_{i, j} a_{i j} \leqslant 2 n$, since by assumption the row sums of $\left(a_{i j}\right)$ are each at most 1 . This implies that the diagonal sum satisfies $\sum_{i} a_{i i} \leqslant 1$, giving the required contradiction.

We next give conditions under which the sum of two diagonal entries of an $n \times n$ stochastic matrix can be bounded from below.

Lemma 5. Let $\left(a_{i j}\right)$ be an $n \times n$ stochastic matrix with diagonal sum $d$, and suppose all transversal sums of $\left(a_{i j}\right)$ lie outside the interval $[u, d)$. Then, for all $i, j$,

$$
a_{i i}+a_{j j}>a_{i j}+a_{j i} \text { implies } a_{i i}+a_{j j}>d-u .
$$

Proof. Suppose $a_{i i}+a_{j j}>a_{i j}+a_{j i}$. Then the positive quantity $a_{i i}+a_{j j}-a_{i j}-a_{j i}$ is the decrease in the diagonal sum caused by transposing rows $i$ and $j$ of the matrix, and so by assumption is greater than $d-u$. We therefore have $a_{i i}+a_{j j} \geqslant a_{i i}+a_{j j}-a_{i j}-a_{j i}>d-u$.

Define an $n \times n$ stochastic matrix $\left(a_{i j}\right)$ to be diagonally ordered if its diagonal entries are in non-increasing order:

$$
a_{11} \geqslant a_{22} \geqslant \ldots \geqslant a_{n n}
$$

We are now ready to prove Theorem 1.

Proof of Theorem 1. We know from (1) and (2) that the result holds for $n=2$ and 3, so we may take $n \geqslant 4$. Suppose, for a contradiction, that $\left(a_{i j}\right)$ is an $n \times n$ stochastic matrix whose transversal sums all lie outside the interval $[1,2]$. Then by Lemma $3,\left(a_{i j}\right)$ has a transversal sum greater than 2 and a transversal sum less than 1 . Let $2+\epsilon$ be the smallest transversal sum greater than 2 , and reorder the rows and columns of $\left(a_{i j}\right)$ so that the summands of this transversal sum occur on the matrix diagonal and so that the matrix is diagonally ordered. By Lemma 5 with $d=2+\epsilon$ and $u=1$,

$$
a_{i i}+a_{j j}>a_{i j}+a_{j i} \text { implies } a_{i i}+a_{j j}>1+\epsilon .
$$

Now the $(n-1) \times(n-1)$ submatrix of $\left(a_{i j}\right)$ formed by deleting the first row and column has diagonal sum $2+\epsilon-a_{11}>1$. Apply Lemma 4 to this submatrix to show that, for some distinct $k>1$ and $\ell>1$,

$$
a_{k k}+a_{\ell \ell}>a_{k \ell}+a_{\ell k} .
$$

We therefore have $a_{k k}+a_{\ell \ell}>1+\epsilon$ by (4), and so

$$
a_{22}+a_{33}>1+\epsilon
$$


since the matrix is diagonally ordered and $k, \ell$ are distinct. Since the diagonal sum of $\left(a_{i j}\right)$ is $2+\epsilon$, we have

$$
a_{i i}+a_{11} \leqslant 2+\epsilon-a_{22}-a_{33} \text { for all } i>3,
$$

and therefore $a_{i i}+a_{11}<1$ for all $i>3$, by (5). Then, since the matrix is diagonally ordered,

$$
a_{i i}+a_{j j}<1 \text { for all } i, j \text { with } i>3,
$$

which in turn implies by (4) that

$$
a_{i i}+a_{j j} \leqslant a_{i j}+a_{j i} \text { for all } i, j \text { with } i>3 \text {. }
$$

We complete the proof by showing that (5) and (6) force the sum of the entries of $\left(a_{i j}\right)$ to be too large. We have

$$
\begin{aligned}
\sum_{i, j} a_{i j} & \geqslant \sum_{i \leqslant 3} a_{i i}+\sum_{i>3} a_{i i}+\sum_{i>3, j \leqslant 3}\left(a_{i j}+a_{j i}\right) \\
& \geqslant(n-2) \sum_{i \leqslant 3} a_{i i}+4 \sum_{i>3} a_{i i}
\end{aligned}
$$

by substitution from (6). Therefore

$$
\begin{aligned}
\sum_{i, j} a_{i j} & \geqslant(n-2) \sum_{i \leqslant 3} a_{i i}+2 \sum_{i>3} a_{i i} \\
& =(n-4) \sum_{i \leqslant 3} a_{i i}+2 \sum_{i} a_{i i} \\
& \geqslant(n-4)(1+\epsilon)+2(2+\epsilon)
\end{aligned}
$$

by (5), using $n \geqslant 4$. Therefore $\sum_{i, j} a_{i j}>n$, which is a contradiction because each row sum of $\left(a_{i j}\right)$ is 1 .

\section{Intervals of length $\frac{n}{n-1}$}

In this section we prove Theorem 2 .

Proposition 6. Let $n \geqslant 4$ and let $\left(a_{i j}\right)$ be a diagonally ordered $n \times n$ stochastic matrix. Suppose the diagonal sum $d$ of $\left(a_{i j}\right)$ satisfies $d \in(1,2]$. Then $\left(a_{i j}\right)$ has a transversal sum lying in the interval $\left[d-\frac{n}{n-1}, d\right)$.

Proof. Suppose, for a contradiction, that no transversal sum of $\left(a_{i j}\right)$ lies in the interval $\left[d-\frac{n}{n-1}, d\right)$. Then by Lemma 5 with $u=d-\frac{n}{n-1}$,

$$
a_{i i}+a_{j j}>a_{i j}+a_{j i} \text { implies } a_{i i}+a_{j j}>\frac{n}{n-1} .
$$


Since $d>1$, Lemma 4 gives

$$
a_{k k}+a_{\ell \ell}>a_{k \ell}+a_{\ell k} \text { for some distinct } k, \ell,
$$

and it follows from (7) that $a_{k k}+a_{\ell \ell}>\frac{n}{n-1}$. Since the matrix is diagonally ordered and $k, \ell$ are distinct, this implies

$$
a_{11}+a_{22}>\frac{n}{n-1}
$$

and

$$
a_{11}>\frac{1}{2} \cdot \frac{n}{n-1}
$$

We now claim that

$$
a_{i i}+a_{j j} \leqslant \frac{n}{n-1} \text { for all distinct } i>1 \text { and } j>1 .
$$

Suppose otherwise, for a contradiction, so that $a_{r r}+a_{s s}>\frac{n}{n-1}$ for some distinct $r>1$ and $s>1$. Since the matrix is diagonally ordered, this gives

$$
a_{22}+a_{33}>\frac{n}{n-1} .
$$

Therefore, for all $i>3$, we have $a_{i i}+a_{11} \leqslant d-a_{22}-a_{33}<\frac{n-2}{n-1}$ because $d \leqslant 2$. Since the matrix is diagonally ordered, we then have

$$
a_{i i}+a_{j j}<\frac{n-2}{n-1} \text { for all } i, j \text { with } i>3
$$

which by (7) implies

$$
a_{i i}+a_{j j} \leqslant a_{i j}+a_{j i} \text { for all } i, j \text { with } i>3 \text {. }
$$

Write

$$
\begin{aligned}
\sum_{i, j} a_{i j} & \geqslant \sum_{i \leqslant 3} a_{i i}+\sum_{i>3, j \leqslant 3}\left(a_{i j}+a_{j i}\right) \\
& \geqslant(n-2) \sum_{i \leqslant 3} a_{i i}+3 \sum_{i>3} a_{i i}
\end{aligned}
$$

by substitution from (12), so that

$$
\begin{aligned}
\sum_{i, j} a_{i j} & \geqslant(n-2) \sum_{i \leqslant 3} a_{i i} \\
& >(n-2) \cdot \frac{3}{2} \cdot \frac{n}{n-1}
\end{aligned}
$$

from (9) and (11). Since $\sum_{i j} a_{i j}=n$ and $n \geqslant 4$, this is a contradiction and proves the claim (10). 
It then follows from (7) that

$$
a_{i i}+a_{j j} \leqslant a_{i j}+a_{j i} \text { for all distinct } i>1 \text { and } j>1 .
$$

Summing over all $i, j$ satisfying $1<i<j$, we find that

$$
(n-2) \sum_{i>1} a_{i i} \leqslant \sum_{1<i<j}\left(a_{i j}+a_{j i}\right) .
$$

Now let $m$ be the largest integer $i$ such that $a_{11}+a_{i i}>\frac{n}{n-1}$. Note that $m \geqslant 2$, by (8). By (7) we have $a_{11}+a_{i i} \leqslant a_{1 i}+a_{i 1}$ for $i>m$, so that

$$
a_{11} \leqslant a_{1 i}+a_{i 1} \quad \text { for } i>m \text {. }
$$

We now show that (13) and (14) force the entries of $\left(a_{i j}\right)$ to be too large. We have

$$
\begin{aligned}
\sum_{i, j} a_{i j} & \geqslant a_{11}+\sum_{i>1} a_{i i}+\sum_{i>m}\left(a_{1 i}+a_{i 1}\right)+\sum_{1<i<j}\left(a_{i j}+a_{j i}\right) \\
& \geqslant(n-m+1) a_{11}+(n-1) \sum_{i>1} a_{i i}
\end{aligned}
$$

by substitution from (13) and (14). Therefore

$$
\begin{aligned}
\sum_{i, j} a_{i j} & \geqslant(n-m+1) a_{11}+(n-1) \sum_{1<i \leqslant m} a_{i i} \\
& >(n-m+1) a_{11}+(n-1)(m-1)\left(\frac{n}{n-1}-a_{11}\right)
\end{aligned}
$$

by definition of $m$ and the diagonal ordering of $\left(a_{i j}\right)$, and so

$$
\sum_{i, j} a_{i j}>n\left(m-1-(m-2) a_{11}\right) .
$$

Since $m \geqslant 2$ and $a_{11} \leqslant 1$, this implies the contradiction $\sum_{i, j} a_{i j}>n$ and so completes the proof.

We now combine Proposition 6 with Theorem 1 to prove Theorem 2.

Proof of Theorem 2. We know from (1) and (2) that the result holds for $n=2$ and 3, so we may take $n \geqslant 4$. Suppose, for a contradiction, that $\left(a_{i j}\right)$ is an $n \times n$ stochastic matrix whose transversal sums all lie outside the interval $\left[u, u+\frac{n}{n-1}\right]$ for some $u \in\left[0, \frac{n-2}{n-1}\right)$. Since this interval contains 1 , by Theorem 1 the matrix $\left(a_{i j}\right)$ therefore has a transversal sum in the interval $\left(u+\frac{n}{n-1}, 2\right]$. Let $d$ be the smallest such transversal sum. Reorder the rows and columns of $\left(a_{i j}\right)$ so that the summands of this transversal sum occur on the matrix diagonal and so that the matrix is diagonally ordered. Then by Proposition $6,\left(a_{i j}\right)$ has a transversal sum lying in the interval $\left[d-\frac{n}{n-1}, d\right)$. By choice of $d$, this gives the required contradiction. 


\section{Acknowledgements}

The authors thank the organisers of the February 2011 14th Coast Combinatorics Conference, University of Victoria, BC, where they first learned of the $n$-card problem, and Prof. Bill Sands for kindly supplying a preprint of the paper [San11].

\section{References}

[BDM09] R. Burkard, M. Dell'Amico, and S. Martello. Assignment Problems. SIAM, Philadelphia, 2009.

[Flo56] M. M. Flood. The travelling-salesman problem. Operations Research, 4: 61-75, 1956 .

[LS05] E. Lenza and B. Sands. A new proof and extension of problem 2620. Crux Mathematicorum with Mathematical Mayhem, 31:319-326, 2005.

[San01] B. Sands. Problem 2620. Crux Mathematicorum with Mathematical Mayhem, 27: 138, 2001. Solution due to M. Bataille in 28: 127-128, 2002.

[San11] B. Sands. Cards, permutations, and sums. Contributions to Discrete Mathematics, 6: 1-19, 2011.

[Zei07] P. Zeitz. The Art and Craft of Problem Solving, 2nd edition, Wiley, 2007. 